\title{
LOCAL BIFURCATION FOR THE FITZHUGH-NAGUMO SYSTEM
}

\author{
Carmen Rocsoreanu \\ Dept. of Math., University of Craiova, A.I. Cuza 13, Romania \\ carmenr@central.ucv.ro \\ Mihaela Sterpu \\ Dept. of Math., University of Craiova, A.I. Cuza 13, Romania \\ mihaelas@central.ucv.ro
}

\begin{abstract}
The 2-D FitzHugh-Nagumo (F-N) system depending on three real parameters $a, b$, and $c$ is considered. It models the electrical potential of the nodal system in the heart. All local bifurcations of equilibria are emphasized in three qualitatively distinct situations concerning the parameter $c(0<c<1, c=1, c>1)$. We found codimension-one bifurcations (saddle-node, Hopf), codimension two bifurcations (Bogdanov-Takens, Bautin, cusp, double-zero with order two symmetry) and a codimension three bifurcation (degenerated Bogdanov-Takens of order two). In addition, some non-generic codimension two bifurcations generated by the coexistence of two codimension one bifurcations are shown. In our study we used the normal form theory [3], [6] and the center manifold theory [2].
\end{abstract}

Keywords: bifurcation, FitzHugh-Nagumo, normal form, center manifold.

\section{Introduction}

Starting with the Hodgkin-Huxley model [5] for nerve axon which consists of a Cauchy problem for a system of four first order ordinary differential equations (ODE), FitzHugh [4] constructed a two dimensional model, describing physiological phenomena that take place in the sinusal node of the heart. This node produces the initiation of the electrical impulse, so it has the role of pace-maker.

The model considered by FitzHugh [4] is the Cauchy problem for the system of ODE's

The original version of this chapter was revised: The copyright line was incorrect. This has been corrected. The Erratum to this chapter is available at DOI: 10.1007/978-0-387-35690-7_44 


$$
\left\{\begin{array}{l}
\frac{d x}{d t}=c\left(x+y+z-\frac{x^{3}}{3}\right), \\
\frac{d y}{d t}=-\frac{1}{c}(x-a+b y)
\end{array}\right.
$$

where $x$ is the electrical potential of the cell membrane, $y$ is an auxiliary variable depending on the refractory period, the parameters $a$ and $b$ are related to the number of channels of the cell membrane which are opened to the $\mathrm{Na}^{+}$and $\mathrm{K}^{+}$ions, $z$ is the injected current and $c \neq 0$ is the relaxation parameter.

For $a=b=z=0$, the well-known Van der Pol system is obtained.

FitzHugh analyzed this model for some particular values of the parameters $a, b, c$ and variable $z$, emphasizing the periods of the cardiac cycle.

In this paper we synthetize our results on local bifurcation around equilibria of the F-N model, for all values of the parameters. The system (1) is reduced to the form

$$
\left\{\begin{array}{l}
\dot{x}=c\left(x+\bar{y}-\frac{x^{3}}{3}\right), \\
\dot{\bar{y}}=-\frac{1}{c}(x-\bar{a}+b \bar{y}),
\end{array}\right.
$$

where $\bar{y}=y+z, \bar{a}=a+b z$. We rename the variables $x, \bar{y}$ as $x_{1}$ and $x_{2}$, and the parameter $\bar{a}$ as $a$, so system (2) becomes

$$
\left\{\begin{array}{l}
\dot{x}_{1}=c\left(x_{1}+x_{2}-x_{1}^{3} / 3\right) \\
\dot{x}_{2}=-\frac{1}{c}\left(x_{1}+b x_{2}-a\right),
\end{array}\right.
$$

As the system (3) is invariant with respect to the transformations $\left(x_{1}, x_{2}, a\right) \rightarrow\left(-x_{1},-x_{2},-a\right)$ and $\left(x_{1}, x_{2}, t, c\right) \rightarrow\left(x_{1}, x_{2},-t,-c\right)$, it is sufficient to investigate the case $c>0, a \geq 0$.

Since its derivation, the F-N system was widely investigated. In more particular or general settings it is a subject of many recent articles. The system has been analyzed within a particular parameter region relevant to physiology [4] and for full parameter space with emphasis on the periodic solutions [1]. A complete study on the phase dynamics and bifurcation of this model for $c>1+\sqrt{3}$ can be found in [7]. In [10], [12] codimension-one, -two, -three local bifurcations for the entire parameter space are determined.

\section{Equilibria and linear stability}

Denote by $D=\left\{\mu=(b, a, c) \in \mathbf{R}^{3}, c \neq 0\right\}$. For $\mu \in D$, denote by $S_{\mu}$ the set of equilibria of system (3) corresponding to $\mu$. This set is defined by the equations

$$
\left\{\begin{array}{l}
x_{1}+x_{2}-\frac{1}{3} x_{1}^{3}=0 \\
x_{1}+b x_{2}-a=0
\end{array}\right.
$$




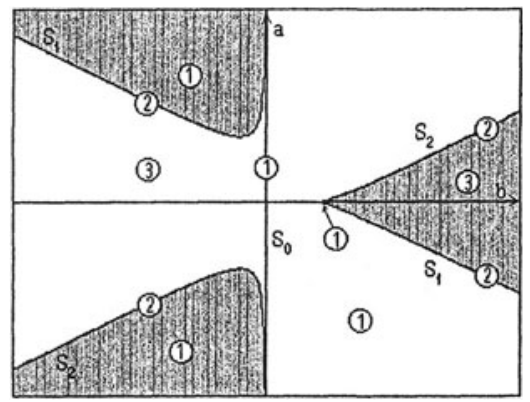

Figure 1. Section in static bifurcation values set with a $c=$ const. plane.

Let $f: \mathbf{R} \rightarrow \mathbf{R}$ be the function defined by $f\left(x_{1}\right)=x_{1}^{3} / 3-x_{1}$.

Remark 1. The correspondence $\left(x_{1}, x_{2}\right) \rightarrow\left(x_{1}, f\left(x_{1}\right)\right.$ in $\mathbf{S}_{\mu}$ is bijective [7]. Therefore, the static bifurcation diagram of (3) is the set

$$
\Sigma=\left\{\left(\mu, x_{1}\right), \quad \mu \in D, \quad \mathbf{x}=\left(x_{1}, x_{2}\right) \in \mathbf{S}_{\mu}\right\} \subset \mathbf{R}^{4} .
$$

For $b=0$, the system (4) possesses a unique solution, $x_{1}=a$, $x_{2}=a^{3} / 3-a$.

For $b \neq 0$, the abscissa $x_{1}$ of an equilibrium satisfies the following equation

$$
x_{1}^{3}-3 x_{1}\left(1-\frac{1}{b}\right)-\frac{3 a}{b}=0 .
$$

Denote by $\Delta=4\left(1-\frac{1}{b}\right)^{3}-\frac{9 a^{2}}{b^{2}}$ the discriminant of (5). Equation (5) possesses (i) three distinct real solutions $\xi_{1}<\xi_{2}<\xi_{3}$ if $\Delta>0$, (ii) two distinct real solutions (one of them being double) if $\Delta=0$, (iii) a unique real solution $\xi_{0}$ otherwise. The solution $\xi_{0}$ is triple for $b=1, a=0$ and single otherwise.

In the parameter space $D$, consider the surfaces

$$
S_{1,2}: a= \pm \frac{2 b}{3}\left(1-\frac{1}{b}\right)^{3 / 2}, b \in(-\infty, 0) \cup[1, \infty)
$$

of parameters to which a double solution of equation (5) corresponds, and $S_{0}: b=0$. The static bifurcation diagram for the F-N system (3) consists of $S_{1} \cup S_{2} \cup S_{0}$. A section with a $c=$ const. plane in the static bifurcation diagram is represented in Figure 1., where the number of equilibria corresponding to each strata is shown.

Remark 2. For $\mu \in S_{1} \cup S_{2}$ we have $\xi_{1}=-2 \sqrt{1-\frac{1}{b}}, \xi_{2}=\xi_{3}=\sqrt{1-\frac{1}{b}}$, for $b<0$, and $\xi_{1}=\xi_{2}=-\sqrt{1-\frac{1}{b}}, \xi_{3}=2 \sqrt{1-\frac{1}{b}}$, for $b \geq 1$. 
Consider a parameter $\mu \in D$ and $\overline{\mathbf{x}}=(\xi, f(\xi)) \in S_{\mu}$. The matrix associated with the linearized system around $\overline{\mathrm{x}}$ reads

$$
M_{\mu}(\overline{\mathbf{x}})=\left(\begin{array}{cc}
c\left(1-\xi^{2}\right) & c \\
-\frac{1}{c} & -\frac{b}{c}
\end{array}\right)
$$

and the characteristic equation is

$$
\lambda^{2}-\lambda\left[c\left(1-\xi^{2}\right)-\frac{b}{c}\right]-b\left(1-\xi^{2}\right)+1=0 .
$$

Denote by $\lambda_{1}, \lambda_{2}$ the corresponding eigenvalues.

The topological type of the hyperbolic equilibria is given by the signature of the determinant $\operatorname{det} M_{\mu}(\overline{\mathbf{x}})=1-b\left(1-\xi^{2}\right)$ and of the trace $\operatorname{tr} M_{\mu}(\overline{\mathbf{x}})=c\left(1-\xi^{2}\right)-b / c$. Consider the sets $\Gamma_{1}$ and $\Gamma_{2}$ from the $\left(b, a, c, x_{1}\right)$ space, defined by

$$
\Gamma_{1}=\left\{\left(\mu, x_{1}\right),\left|x_{1}\right|=\Gamma_{1}(b, c), a \geq 0, c>0, b \leq c^{2}\right\},
$$

and

$$
\Gamma_{2}=\left\{\left(\mu, x_{1}\right),\left|x_{1}\right|=\Gamma_{2}(b), a \geq 0, c>0, b \in(-\infty, 0) \cup[1, \infty)\right\},
$$

where $\Gamma_{1}(b, c)=\sqrt{1-b / c^{2}}$ and $\Gamma_{2}(b)=\sqrt{1-1 / b}$. With these notations we have $\operatorname{det} M_{\mu}(\overline{\mathbf{x}})=0$ if and only if $(\mu, \xi) \in \Gamma_{2}$ and $\operatorname{tr} M_{\mu}(\overline{\mathbf{x}})=0$ if and only if $(\mu, \xi) \in \Gamma_{1}$.

In the non-hyperbolic case, if $\operatorname{Re}\left(\lambda_{1}\right)$ and/or $\operatorname{Re}\left(\lambda_{2}\right)$ vanish, nonlinear terms are to be considered in order to determine the topological type of $\overline{\mathbf{x}}$.

Sections in the sets $\Gamma_{1}$ and $\Gamma_{2}$ with a plane $c=$ const., $a=$ const. are given in Figures 2, 3, 4, for a $c<1, c=1$, and $c>1$, respectively. The distinction was made by the number of intersections $\Gamma_{1} \cap \Gamma_{2}$ in such a plane. Namely, for $c<1, \Gamma_{1} \cap \Gamma_{2}$ consists of two points (denoted by $Q_{1}, Q_{4}$ ), for $c=1$, of three points (denoted by $Q_{1}, Q_{4}, Q$ ) and for $c>1$ of four points $\left(Q_{1,2,3,4}\right)$. In Figures 2, 3, 4, we have $\operatorname{tr} M_{\mu}(\overline{\mathbf{x}})>0$ and $\operatorname{det} M_{\mu}(\overline{\mathbf{x}})>0$ in region I, $\operatorname{det} M_{\mu}(\overline{\mathbf{x}})<0$ in region II, and $\operatorname{tr} M_{\mu}(\overline{\mathbf{x}})<0$ and $\operatorname{det} M_{\mu}(\overline{\mathbf{x}})>0$ in region III. Consequently, this corresponds to the regions where the sign of $\operatorname{Re} \lambda_{1,2}$ is preserved.

Remark 3. In Fig. 2, equilibria $\overline{\mathbf{x}}=(\xi, f(\xi)) \in S_{\mu}$ with $(\mu, \xi) \in \Gamma_{1}$ situated between $Q_{1}$ and $Q_{4}$ have $\operatorname{Re} \lambda_{1}=\operatorname{Re} \lambda_{2}=0, \lambda_{1,2} \in \mathbf{C}-\mathbf{R}$, so these equilibria are candidates for Hopf bifurcation. The same conclusion is valid for points situated between $Q_{1}$ and $Q$ or $Q_{4}$ and $Q$ on $\Gamma_{1}$ in Fig. 3 , and for points between $Q_{1}$ and $Q_{2}$ or $Q_{3}$ and $Q_{4}$ on $\Gamma_{1}$ in Fig. 4.

Remark 4. In Figures 2, 3, 4, for equilibria $\overline{\mathbf{x}}=(\xi, f(\xi)) \in S_{\mu}$ with $(\mu, \xi) \in \Gamma_{2}$ we have $\operatorname{det} M_{\mu}(\overline{\mathbf{x}})=0$, hence at least one of the eigenvalues $\lambda_{1,2}$ is zero. Such equilibria are candidates for saddle-node bifurcation. 


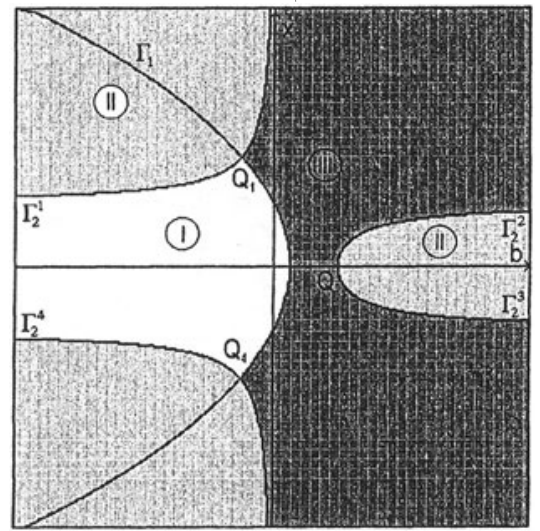

Figure 2. The regions in the $\left(b, x_{1}\right)$ plane where the sign of $\operatorname{Re} \lambda_{1,2}$ is preserved, if $c \in(0,1):(\mathrm{I}) \operatorname{Re} \lambda_{1}>0$, $\operatorname{Re} \lambda_{2}>0$; (II) $\operatorname{Re} \lambda_{1}<0$, $\operatorname{Re} \lambda_{2}>0$, (III) $\operatorname{Re} \lambda_{1}<0$, Re $\lambda_{2}<0$.

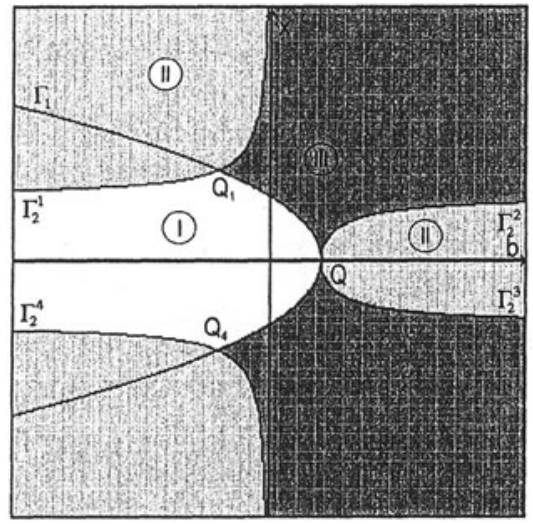

Figure 3. The regions in the $\left(b, x_{1}\right)$ plane where the sign of $\operatorname{Re} \lambda_{1,2}$ is preserved, if $c=1$ : (I) $\operatorname{Re} \lambda_{1}>0, \operatorname{Re} \lambda_{2}>0$; (II) $\operatorname{Re} \lambda_{1}<0, \operatorname{Re} \lambda_{2}>0$, (III) $\operatorname{Re} \lambda_{1}<0$, Re $\lambda_{2}<0$.

Remark 5. Equilibria $\overline{\mathbf{x}}=(\xi, f(\xi)) \in S_{\mu}$ with $(\mu, \xi) \in Q_{i}, i=1,4$, have $\lambda_{1}=\lambda_{2}=0$, so they are candidates for a double zero bifurcation.

The projection of the set $\Sigma \cap \Gamma_{1}$ on the $(b, a, c)$ space is the set $H_{1} \cup H_{2}$, where

$$
H_{1,2}: a= \pm \frac{1}{3 c^{3}}\left(3 c^{2}-b^{2}-2 b c^{2}\right) \sqrt{c^{2}-b} .
$$

Only the branches of $\Gamma_{1}$ with $b \in(-c, c)$ for $c>1$ or $b \in\left(-c, c^{2}\right]$ for $c \leq 1$ separates regions with different signs of Re $\lambda_{1,2}$, so only these branches will be considered in the following.

The projection of $\Sigma \cap \Gamma_{2}$ on the $(b, a, c)$ space is the set $S_{1} \cup S_{2}$, given by (7). 


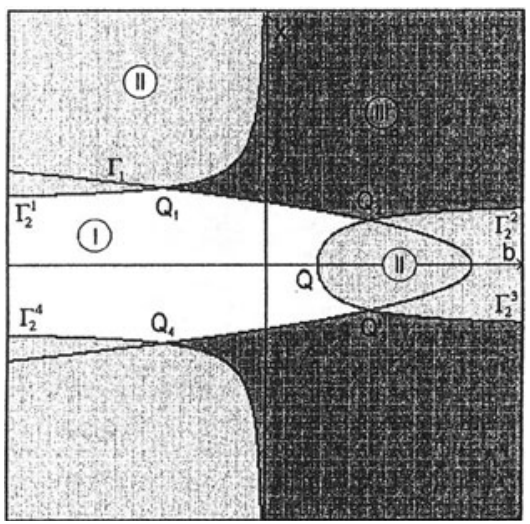

Figure 4. The regions in the $\left(b, x_{1}\right)$ plane where the sign of $\operatorname{Re} \lambda_{1,2}$ is preserved, if $c>1$ : (I) $\operatorname{Re} \lambda_{1}>0$, $\operatorname{Re} \lambda_{2}>0$; (II) $\operatorname{Re} \lambda_{1}<0$, $\operatorname{Re} \lambda_{2}>0$, (III) $\operatorname{Re} \lambda_{1}<0$, Re $\lambda_{2}<0$.

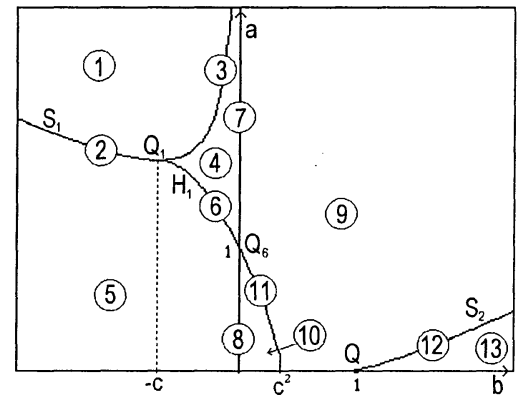

Figure 5. Section in the surfaces $H_{1,2} \cup S_{1,2}$ with a $c=$ const. plane, $c \in(0,1)$.

The intersection of $H_{1} \cup H_{2}$ with a $c=$ const. plane depends significantly on $c$. We found three typical cases, corresponding to $c<1$, $c=1, c>1$, respectively. They are qualitatively illustrated in Figures $5,6,7$. The curves of intersection of the surfaces $H_{1}, H_{2}, S_{1}, S_{2}$ with a $c=$ const. plane where denoted in the same way as the corresponding surfaces. In every plane $c=$ const., at the intersection points $Q_{i}$, $i=1,4$, the curves $H_{1} \cup H_{2}$ and $S_{1} \cup S_{2}$ cuts tangentially. Consider also the points $Q_{6}=H_{1} \cap S_{0}$ and, for $c>1, Q_{0}=H_{1} \cap H_{2}, Q_{5}=H_{1} \cap S_{2}$.

Taking into account the sign of $\operatorname{Re} \lambda_{1,2}$ established in Figures 2, 3, 4, in Figures 5, 6, 7 the type of hyperbolic equilibria is emphasized. Thus, we have:

- for $(b, a)$ situated in region 1: a saddle $\times$;

- for $(b, a)$ situated in region 4: two saddles and an attractor $\times, \bullet, \times$;

- for $(b, a)$ situated in region 5: two saddles and a repulsor $\times, \circ, \times$;

- for $(b, a)$ situated in region 7,9 : an attractor $\bullet$; 


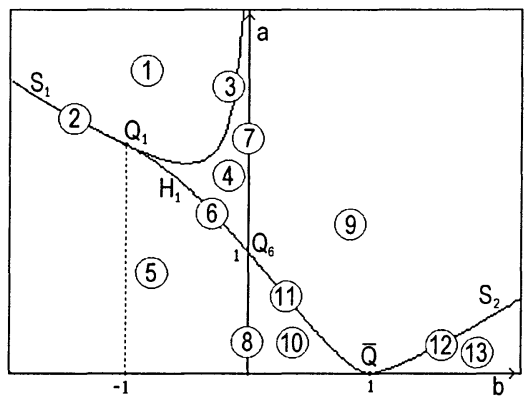

Figure 6. Section in the surfaces $H_{1,2} \cup S_{1,2}$ with a $c=$ const. plane, $c=1$.
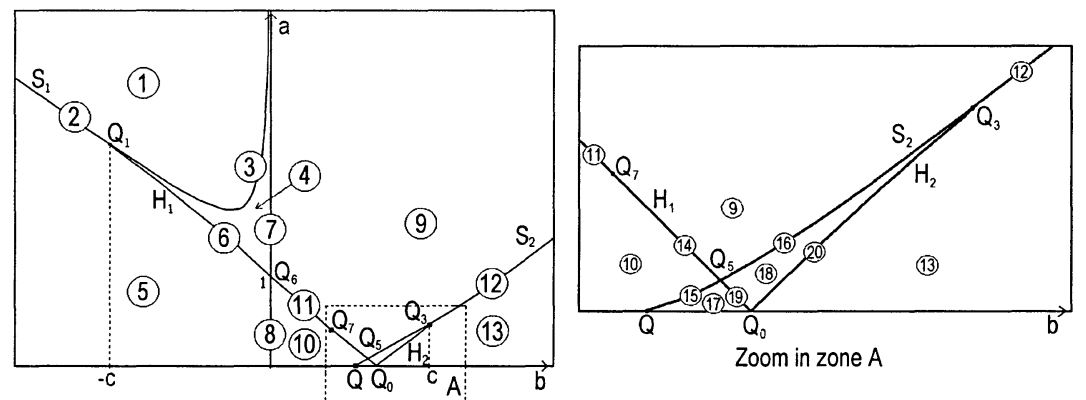

Figure 7. Section in the surfaces $H_{1,2} \cup S_{1,2}$ with a $c=$ const. plane, $c>1$.

- for $(b, a)$ situated in region 8, 10: a repulsor $\circ$;

- for $(b, a)$ situated in region 13: two attractors and a saddle $\bullet, \times, \bullet$;

- for $(b, a)$ situated in region 17: two repulsors and a saddle $\circ, \times, \circ$;

- for $(b, a)$ situated in region 18: a repulsor, a saddle and an attractor $\circ, \times, \bullet$.

In these regions the system possesses only hyperbolic equilibria. We also found the topological type (hyperbolic or not) for the other regions. Namely, we have:

- for $(b, a)$ situated in the regions 2,3 or at $Q_{1}$ : a saddle and a non-hyperbolic equilibrium $\times, \square$;

- for $(b, a)$ situated at $Q_{5}$ : two non-hyperbolic equilibria $\square, \square$;

- for $(b, a)$ situated in region 6: two saddles and a non-hyperbolic equilibrium $\times, \square, \times$;

- for $(b, a)$ situated in regions 11,14 , at $Q_{6}$ or at $Q$ : a non-hyperbolic equilibrium $\square$;

- for $(b, a)$ situated in region 15: a non-hyperbolic equilibrium and a repulsor $\square$, o; 
- for $(b, a)$ situated in regions 12,16 or at $Q_{3}:$ a non-hyperbolic equilibrium and an attractor $\square, \bullet$;

- for $(b, a)$ situated at $Q_{0}$ : two non-hyperbolic equilibria and a saddle $\square, \times, \square$;

- for $(b, a)$ situated in region 19: a repulsor, a saddle and a nonhyperbolic equilibrium $\circ, \times, \square$;

- for $(b, a)$ situated in region 20: a non-hyperbolic equilibrium, a saddle and an attractor $\square, \times, \bullet$.

The topological type of the non-hyperbolic equilibria is established in Section 2.

\section{Local bifurcation}

For each non-hyperbolic equilibria we found the topological normal form. This allowed us to establish the topological type of non-hyperbolic equilibria and the local bifurcation generated by their presence.

\subsection{Saddle-node bifurcation}

Consider $\mu \in S_{1} \cup S_{2}-\{(b, a, c), b= \pm c\}$ and $\overline{\mathbf{x}}=(\xi, f(\xi)) \in S_{\mu}$ with $\xi^{2}=1-\frac{1}{b}$. Thus, for the equilibrium $\overline{\mathbf{x}}$ we have $\lambda_{1}=\frac{c^{2}-b^{2}}{b c} \neq 0$ and $\lambda_{2}=0$

First, assume $b \neq 1$. Hence $\xi \neq 0$. Using certain changes of coordinates [7], [11], system (3) is topologically equivalent with the following system

$$
\left\{\begin{array}{l}
\dot{y}_{1}=\lambda_{1} y_{1}+\frac{2 c^{2} \xi}{\lambda_{1}} y_{1} y_{2}+O\left(|\mathbf{y}|^{3}\right), \\
\dot{y}_{2}=-\frac{\xi b^{2}}{\lambda_{1}} y_{2}^{2}+O\left(|\mathbf{y}|^{3}\right)
\end{array}\right.
$$

which is the normal form for a non-degenerated saddle-node equilibrium. The bifurcation corresponding to such parameters is a codimension-one saddle-node bifurcation. The local dynamics around such an equilibrium is deduced using the center manifold theorem. As the center manifold for system (12) is given by $y_{1}=0$, up to third order terms, the flow on the center manifold is determined by

$$
\dot{y}_{2}=-\frac{\xi b^{2}}{\lambda_{1}} y_{2}^{2}+O\left(\left|y_{2}\right|^{3}\right) .
$$

Thus, for $b \in(-\infty,-c) \cup(\min \{1, c\}, c)$ the equilibrium $\overline{\mathbf{x}}$ possesses three repulsive directions and an attractive one, while for $b \in(-c, 0) \cup$ $(\max \{1, c\}, \infty)$ the equilibrium $\overline{\mathbf{x}}$ possesses three attractive directions and a repulsive one.

If $b=1$ then $a=0$ and $\xi=0$ is the abscissa of the triple equilibrium. In this case, if $c \neq 1$ we have $\lambda_{1} \neq 0$. Using appropriate transformations 
[7], [12], system (3) is written as

$$
\left\{\begin{array}{l}
\dot{y}_{1}=\lambda_{1} y_{1}-\frac{c^{2}}{\lambda_{1}} y_{1} y_{2}^{2}+O\left(|\mathbf{y}|^{4}\right) \\
\dot{y}_{2}=\frac{1}{3 \lambda_{1}} y_{2}^{3}+O\left(|\mathbf{y}|^{4}\right)
\end{array}\right.
$$

which represents the topological normal form for a simple degenerated saddle-node equilibrium. The bifurcation corresponding to these parameters is a cusp bifurcation.

The flow on the center manifold $y_{1}=0$ is given by [10]

$$
\dot{y}_{2}=\frac{1}{3 \lambda_{1}} y_{2}^{3}+O\left(|\mathbf{y}|^{4}\right) \text {. }
$$

Therefore, the equilibrium $\overline{\mathbf{x}}$ is a weakly attractive degenerated saddlenode if $c<1$ and a weakly repulsive degenerated saddle-node if $c>1$.

\subsection{Hopf bifurcation}

Consider $\mu \in H_{1} \cup H_{2}$ such that $b \in\left(-c, c^{2}\right]$ as $c<1$ and $b \in(-c, c)$ as $c \geq 1$. In these cases the $\mathrm{F}-\mathrm{N}$ system possesses an equilibrium $\overline{\mathbf{x}}=(\xi, f(\xi)) \in S_{\mu}$ with $\xi^{2}=1-\frac{b}{c^{2}}$, for which $\lambda_{1,2} \in \mathbf{C}-\mathbf{R}$ and $\operatorname{Re} \lambda_{1}=\operatorname{Re} \lambda_{2}=0$.

Using a sequence of transformations and making the computations in the complex field [7], system (3) is reduced, around a point $\mathbf{x}$ near the equilibrium $\overline{\mathbf{x}}$, to its normal form:

$$
\frac{d w}{d \theta}=(\gamma(\alpha)+i) w+l_{1}(\alpha) w^{2} \bar{w}+l_{2}(\alpha) w^{3} \bar{w}^{2}+O\left(|w|^{6}\right),
$$

where $l_{1}(\alpha)$ and $l_{2}(\alpha)$ are the first and the second Liapunov coefficients, respectively, and were computed in [7], [9]. For $\alpha=\mu$ and $\mathbf{x}=\overline{\mathbf{x}}$ we have $l_{1}=\frac{-b^{2}+2 b c^{2}-c^{2}}{2 c{\sqrt{1-\frac{b^{2}}{c^{2}}}}^{3}}$ and $l_{2}=\frac{10 b^{2}\left(b-c^{2}\right)}{144 c{\sqrt{1-\frac{b^{2}}{c^{2}}}}^{3}}$ if $l_{1}=0$ [11].

Note that $l_{1}=0$ iff $b=b^{*}=c^{2}-c \sqrt{c^{2}-1}$. Consequently, (i) if $c \leq 1$, or $c>1$ and $b \neq b^{*}$, then $l_{1} \neq 0$, hence $\overline{\mathbf{x}}$ is a non-degenerated Hopf equilibrium and a codimension-one Hopf bifurcation takes place; (ii) if $c>1$ and $b=b^{*}$ then $l_{1}=0$ and $l_{2} \neq 0$, hence $\overline{\mathbf{x}}$ is a degenerated Hopf equilibrium of order two.

Denote by $Q_{7}\left(b^{*}, a^{*}, c\right)$ the corresponding point of $H_{1}$. In the last case, a Bautin bifurcation takes place and from the point $Q_{7}$ emerge the curves of non-hyperbolic limit cycle bifurcation values, which is approximated asymptotically, near $Q_{7}$, by the curve $B a$ defined by the equation

$$
l_{2} l_{1}^{2}+4 \frac{\operatorname{Re} \lambda}{\operatorname{Im} \lambda}=0
$$

deduced in [7], [9]. 


\subsection{Bogdanov-Takens bifurcation}

Consider $\mu \in\left(H_{1} \cup H_{2}\right) \cap\left(\dot{S}_{1} \cap S_{2}\right)$, with $|b|=c$, and $\overline{\mathbf{x}}=(\xi, f(\xi)) \in S_{\mu}$ with $\xi^{2}=1-\frac{1}{b}$. The corresponding points in Figures 4, 5, 6 are denoted by $Q_{1-4}$. In this case $\lambda_{1}=\lambda_{2}=0$.

Using appropriate transformations, the normal form of (3) at the point $\mu$ reads

$$
\left\{\begin{array}{l}
\dot{y}_{1}=y_{2} \\
\dot{y}_{2}=-b \xi y_{1}^{2}-2 c \xi y_{1} y_{2}-\frac{b}{3} y_{1}^{3}-c y_{1}^{2} y_{2}
\end{array}\right.
$$

If $b \neq 1$, then $\xi \neq 0$, hence $\overline{\mathbf{x}}$ is a non-degenerated Bogdanov-Takens equilibrium. Around such an equilibrium, system (3) is topologically equivalent with the following

$$
\left\{\begin{array}{l}
\dot{y}_{1}=y_{2} \\
\dot{y}_{2}=\beta_{1}+s \beta_{2} y_{2}+y_{1}^{2}-y_{1} y_{2}
\end{array}\right.
$$

where the coefficients $\beta_{1}=-\frac{16 c^{4} \bar{\xi}}{b^{3}}\left[a+(b-1) \bar{\xi}-b \bar{\xi}^{3} / 3\right], \beta_{2}=2\left(c^{2} / b^{2}-1\right)$ are given in [11] and $s=-1$ at $Q_{1}, Q_{4}$, and $s=1$ at $Q_{2}, Q_{3}$, while $\bar{\xi}=s \sqrt{(1-1 / b)}$. Consequently, the F-N system (3) exhibits at $Q_{1-4}$ codimension-two Bogdanov-Takens bifurcations. In addition, in every plane $c=$ const., at the points $Q_{i}$ emerge the curves $H L_{i}$ corresponding to homoclinic bifurcation values [8], [11]. The equations for $H L_{1,3}$ are approximated by

$$
H L_{ \pm}: a=\frac{1.47\left(1-b^{2} / c^{2}\right)^{2}-2(b-1)^{2}}{3 b \bar{\xi}} .
$$

If $b=1$, then $\xi=0$ and $\overline{\mathbf{x}}$ is a degenerated Bogdanov-Takens equilibrium of order two. Around this equilibrium, system (3) is topologically equivalent with the following

$$
\left\{\begin{array}{l}
\dot{x}_{1}=x_{2} \\
\dot{x}_{2}=\varepsilon_{1}+\varepsilon_{2} x_{1}+\varepsilon_{3} x_{2}-x_{1}^{3}-x_{1}^{2} x_{2}
\end{array}\right.
$$

where

$$
\varepsilon_{1}=\frac{9 \sqrt{3} c^{3} a}{b^{2} \sqrt{b}}, \varepsilon_{2}=\frac{9 c^{2}(b-1)}{b^{2}}, \varepsilon_{3}=\frac{3\left(c^{2}-b\right)}{b} .
$$

Consequently, a codimension three bifurcation takes place around this point.

\subsection{Double-zero with symmetry of order two bifurcation}

In the following we restrict to the case $a=0$. Consider $\mu=(1,0,1)$. Then the F-N system (3) possesses a triple equilibrium $\overline{\mathbf{x}}=(0,0)$. 
Around $(\mu, \overline{\mathbf{x}})$, with $\mu$ situated in the $a=0$ plane, system (3) is topologically equivalent with the following [13]

$$
\left\{\begin{array}{l}
\dot{y}_{1}=y_{2} \\
\dot{y}_{2}=\varepsilon_{2} y_{1}+\varepsilon_{3} y_{2}-y_{1}^{3}-y_{1}^{2} y_{2}
\end{array}\right.
$$

which is a normal form for a double zero with symmetry of order two bifurcation. Denote by $\bar{Q}(1,1)$ the point corresponding to $\mu$ in the $a=0$ plane. Consequently, from $\bar{Q}$ the following bifurcation curves emerge [11]:

- $H L=\left\{(b, c), b>1, \frac{3}{5 b^{2}}\left[7 c^{2} b-12 c^{2}+5 b^{2}\right]+O\left(\varepsilon_{2}^{3 / 2}\right)=0\right\}$, corresponding to double homoclinic bifurcation values,

$-B=\left\{(b, c), b>1, \frac{3}{b^{2}}\left[\left(c_{0}-1\right) c^{2} b-c_{0} c^{2}+b^{2}\right]+O\left(\varepsilon_{2}^{3 / 2}\right)=0\right\}$, where $c_{0} \approx 0.752$, corresponding to non-hyperbolic limit cycle bifurcation values,

- $H=\left\{(b, c), b>1,2 c^{2} b-3 c^{2}+b^{2}=0\right\}$, corresponding to two simultaneously Hopf bifurcation values,

- $R^{+}=\left\{(b, c), b=1, c^{2}-b>0\right\}, R^{-}=\left\{(b, c), b=1, c^{2}-b<0\right\}$, corresponding to cusp bifurcation values,

- $H^{\prime}=\left\{(b, c), b<1, c^{2}-b=0\right\}$, corresponding to Hopf bifurcation values.

\section{Concluding remarks}

The analysis in Section 2 allowed us to completely determine the topological type of non-hyperbolic equilibria emphasized in Section 1.

Thus, the non-hyperbolic equilibria corresponding to parameter values situated on $S_{1} \cup S_{2}$, in regions 2, 15, 16 in Figures 5, 6, 7, are partially repulsive saddle-nodes, while for parameter values situated in regions 3 , 12 , they are partially attractive saddle-nodes.

The non-hyperbolic equilibria corresponding to parameter values situated on $H_{1} \cup H_{2}$, in regions 6,11 or at $Q_{6}$ in Figures $5,6,7$, are slowly attractive Hopf equilibria, while for parameter values situated in regions $14,18,20$, they are slowly repulsive Hopf equilibria.

The non-hyperbolic equilibria corresponding to parameter values situated at $Q_{1}$ or $Q_{3}$ are non-degenerated Bogdanov-Takens equilibria, while for parameter values situated at $Q_{7}$ correspond degenerated Hopf (Bautin) equilibria.

In addition, non-generic codimension-two bifurcations take place at $Q_{5}$ and $Q_{0}$ due to the coexistence of two different non-hyperbolic equilibria, namely a partially repulsive saddle-node and a slowly repulsive Hopf equilibrium at $Q_{5}$, and two slowly repulsive Hopf equilibria at $Q_{0}$. 
Finally, the non-hyperbolic equilibria corresponding to parameter values situated at $Q$ are degenerated saddle-node (cusp) equilibria, which are slowly attractive for $c<1$ and slowly repulsive for $c>1$. For $c=1$, the point $Q=\bar{Q}$ corresponds to degenerated Bogdanov-Takens of order two equilibrium, consequently to a codimension-three bifurcation. This is the unique generic codimension-three local bifurcation exhibited by the FitzHugh-Nagumo model (3).

\section{Acknowledgment}

The first author was partially supported by the grant MEC 260 44/2001.

\section{References}

[1] Barnes B., Grimshaw R. Analytical and numerical studies on the Bonhoeffer van der Pol system, J. Aust. Math. Soc. Series B, 38, (1995), 427-453.

[2] Carr J. Application of Center Manifold Theory, Springer, New York, (1981).

[3] Chow, S.N., Li, C., Wang, D. Normal forms and bifurcation of Planar Vector Fields, Cambridge University Press, 1994.

[4] FitzHugh, R. Impulses and physiological states in theoretical models of nerve membrane, Biophysical J., 1, 1961, pp. 445-466.

[5] Hodgkin A.L., Huxley A.F. A quantitative description of membrane current and its application to conduction and excitation in nerve, J. Physiol., 117, (1952), 500-544.

[6] Kuznetsov, Yu. Elements of applied bifurcation theory, Springer, New York, 1995.

[7] Rocşoreanu, C., Georgescu, A., Giurgiţeanu, N., The FitzHugh-Nagumo Model. Bifurcation and Dynamics, Kluwer Academic Publishers, Dordrecht, 2000.

[8] Rocşoreanu C., Giurgiţeanu, Georgescu A., Degenerated Hopf bifurcation in the FitzHugh-Nagumo system. 1. Bogdanov-Takens bifurcation, Ann. Univ. Timisoara, XXXV, (2), 1997, pp.285-298.

[9] Rocşoreanu C., Giurgiţeanu, Georgescu A., Degenerated Hopf bifurcation in the FitzHugh-Nagumo system. 1. Bautin bifurcation, Revue D'analyse numérique et de théorie de l'approximation, 29, 1, 2000, pp.97-109.

[10] Sterpu, M., Georgescu A., Codimension three bifurcations for the FitzHughNagumo system, Mathematical Reports, 3 (53),3, 2001 (in print).

[11] Sterpu, M., Dynamics and bifurcation for two generalized Van der Pol models, Univ. of Piteşti Publisher, 2001 (romanian).

[12] Sterpu, M, Saddle-node bifurcation in the FitzHugh-Nagumo Model, Mathematical Reports, 4 (54), 3, 2002 (in print).

[13] Sterpu, M, A double-zero with symmetry of order two bifurcation in the FitzHugh-Nagumo model Ann. of Univ. of Craiova, XXIX, 2002 (in print). 